\title{
HISTOMORFOMETRIA DO MIOCÁRDIO DE RATOS DIABÉTICOS TRATADOS COM AZADIRACHTA INDICA, A. JUSS
}

\author{
HISTOMORPHOMETRY OF THE MYOCARDIUM OF DIABETIC RATS TREATED WITH \\ AZADIRACHTA INDICA, A. JUSS.
}

\author{
G. G. RIVERA ${ }^{1}$, T. B. CORSINI ${ }^{1}$, F. H. F. D. ANGELIS ${ }^{2}$, , \\ S. M. BARALDI ARTONI ${ }^{2}$, L. AMOROSO ${ }^{2}$, M. R. PACHECO ${ }^{2}$
}

\begin{abstract}
RESUMO
Esse estudo avaliou a histomofometria dos cardiomiócitos tratados com extrato aquoso e hidroalcoólico de Azadirachta indica a $10 \%$ e da estreptozootocina $6 \mathrm{CH}$ em ratos com Diabetes mellitus induzido por estreptozootocina. Vinte ratos Wistar albinos machos foram distribuídos aleatoriamente em cinco grupos, sendo um grupo controle. Após indução do diabetes com estreptozootocina, os animais foram tratados durante 30 dias da seguinte forma: grupo diabético, sem tratamento; extrato aquoso de Azadirachta indica a 10\%; extrato hidroalcoólico de Azadirachta indica a $10 \%$ e um grupo tratado com estreptozootocina $6 \mathrm{CH}$. No $31^{\circ}$ dia, os animais foram eutanasiados e o coração foi fixado em solução de Bouin e as secções semisseriadas foram coradas pela técnica da hematoxilina-eosina (HE) e PAS/Hematoxilina. Observou-se eosinofilia no citoplasma dos cardiomiócitos pela coloração com HE, o que demonstra a presença de glicogênio no citoplasma dos cardiomiócitos em todos os grupos. Além disso, observou-se que os grupos tratados apresentaram semelhantes áreas e diâmetros, entretanto esses parâmetros diferiram do controle. A maioria dos parâmetros morfométricos nos cardiomiócitos apresentou valores inferiores nos animais diabéticos e em todos grupos tratados. O fitoterápico com Azadirachta indica e homeopático com estreptozootocina 6CH utilizados no Diabetes mellitus induzido pela estreptozootocina não promove modificações morfométricas notáveis nos cardiomiócitos no período de até 30 dias de tratamento.
\end{abstract}

PALAVRAS-CHAVE: Coração. Diabetes mellitus. Estreptozootocina 6CH. Fator forma. Nim.Wistar.

\section{SUMMARY}

This study evaluates the histomorphometry of cardiomyocytes treated with aqueous and hydro-alcoholic extracts of $10 \%$ Azadirachta indica and 6CH streptozotocin in rats with streptozotocin-induced Diabetes mellitus. A total of 20 male albino Wistar rats were divided into five groups. After inducing diabetes by streptozotocin injection, 16 animals were treated for 30 days as follows: 1) diabetic-induced group was not treated; 2) group with $10 \%$ Azadirachta indica aqueous extract; 3) group with 10\% Azadirachta indica hydro-alcoholic extract; and, 4) group with 6CH streptozotocin. On day $31^{\text {st }}$, the animals were killed and the heart was fixed in Bouin's solution and histological sections were stained with Hematoxylin-Eosin (HE) and PAS/Hematoxylin. HE staining showed eosinophilia in the cytoplasm of cardiomyocytes, demonstrating the presence of glycogen in the cytoplasm of cardiomyocytes. Furthermore, it was observed that all treatment groups showed areas and diameters similars, however these parameters differ from the control. Cytoplasmic and nuclear morphometry showed that the control group had the highest values for almost all parameters measured in cardiomyocytes compared to other groups. The most of morphometric parameters in cardiomyocytes showed lower values in diabetic animals and in all treated groups. Azadirachta indica and streptozotocin $6 \mathrm{CH}$ used in Diabetes mellitus induced by streptozotocin not submitted morphological changes in cardiomyocytes.

KEY-WORDS: Diabetes mellitus. Estreptozotocin 6CH. Heart. Form factor. Neem. Wistar.

\footnotetext{
${ }^{1}$ Residente - Universidade Estadual Paulista " Júlio de Mesquita Filho" da Faculdade de Ciências Agrárias e Veterinárias, UNESP/ FCAV, Campus Jaboticabal. gabigrivera@hotmail.com, talitabeani@ hotmail.com

${ }^{2}$ Professora Doutora - Universidade Estadual Paulista " Júlio de Mesquita Filho" da Faculdade de Ciências Agrárias e Veterinárias, UNESP/ FCAV, Campus Jaboticabal. floradan@ig.com.br, smbart@ fcav.unesp.br, lizandra.amoroso@ gmail.com, rpacheco@fcav.unesp.br
} 


\section{INTRODUÇÃO}

A Azadirachta indica pertence à família Meliaceae (VILELA, 2008). Pesquisas recentes estão crescendo no Brasil e no mundo (BRASIL, 2013). Na literatura, têm sido descritas atividades antissépticas, curativas, anti-úlcera, antiinflamatória, antifertilidade, hipolipidêmica e hepatoprotetora (MOSSINI e KEMMELMEIER, 2005). A Diabetes mellitus (DM) está associada à cardiomiopatia, caracterizada por alterações no metabolismo e desempenho cardíaco. Em ratos Wistar diabéticos aloxânicos, após emprego da reação histoquímica pelo Ácido Periódico de Schiff (PAS), evidenciamos acúmulo acentuado de glicogênio nos cardiomiócitos (GOMES, et al., 2008). A alteração predominante que ocorre no metabolismo cardíaco no DM é a supressão da utilização de glicose e o uso excessivo de ácidos graxos associados ao estoque intracelular de lipídios, segundo Ding e Rodrigues (2006). A maior intensidade da reação de PAS no miocárdio de ratos diabéticos pode ser decorrente do glicogênio poupado nessa musculatura. Segundo Jacob et al. (1999), o excesso de glicogênio pode ser maléfico para o órgão ocasionando a doença de Pompe. Entretanto, Satyanarayana et al. (2015) relataram que o nim promove a expressão de moléculas sinalizadoras da insulina e no transportador de glicose citosólica (GLUT4) o que aumenta a oxidação do músculo esquelético favorecendo a utilização de glicose. Silva et al (2013) verificaram que o treinamento físico aeróbico, por oito semanas, reduziu a área citoplasmática, as alterações mitocondriais e o conteúdo de colágeno em miócitos ventriculares dos animais diabéticos. Apesar das suas atividades terapêuticas terem sido comprovadas com trabalhos científicos, não foram encontrados dados na literatura que relatam sua ação benéfica em relação ao Diabetes Mellitus. Acreditamos também que as análises histomorfométricas dos cardiomiócitos trarão contribuições científicas para estabelecer padrões morfométricos que possam ser comparáveis às possíveis alterações que o Diabetes mellitus venha causar nesta célula além do efeito benéfico da terapia com extratos de Azadirachta indica A. Juss. e estreptozootocina $(6 \mathrm{CH})$ sobre esta enfermidade. Dessa forma, avaliamos a histomorfometria das fibras musculares com a utilização de tratamentos embasados na Azadirachta indica, A. Juss e estreptozootocina $(6 \mathrm{CH})$, em ratos com Diabetes mellitus.

\section{MATERIAL E MÉTODOS}

O trabalho foi aprovado pela Comissão de Ética no Uso de Animais (CEUA), com protocolo ${ }^{\circ}$ 025471-05. Foram preparados dois extratos de Azadirachta indica A. Juss., um aquoso e outro etanólico, com as folhas do "nim" cultivadas e fornecidas pela EMBRAPA-CNPAF (Goiânia, Go), que previamente passaram por processo de estabilização à sombra, em ambiente coberto e ventilado. Após secagem e posterior trituração das folhas, preparou-se extrato etanólico a $70 \%(\mathrm{p} / \mathrm{p})$ por percolação, até esgotamento do ativo, numa velocidade de 8 gotas por minuto, e em seguida, esse foi concentrado até a obtenção de um extrato de consistência pastosa. O outro foi um extrato aquoso, utilizando a água destilada para realizar a percolação até esgotamento do ativo, nas mesmas condições anteriores, e posteriormente, o extrato aquoso foi liofilizado. A relação da droga/insumo inerte foi 1:10 $(\mathrm{p} / \mathrm{v})(10 \%)$. As ultra-alta diluições foram preparadas em sistemas dinamizados (estreptozootocina $6 \mathrm{CH}$ ) usando estreptozootocina (Sigma, SP) como ponto de partida e soluções de etanol de diferentes concentrações $(30 \%$ ou $70 \%)$ como veículo. As diluições por um fator de 100 foram realizadas mecanicamente ${ }^{3}$. Foram utilizados 20 ratos albinos, machos, da raça Wistar com peso entre 200 e 250 gramas, provenientes do Biotério Central do Campus de Botucatu - UNESP. Estes foram divididos em cinco grupos de quatro animais cada, adaptados em caixas no biotério, com temperatura controlada e ciclo de claroescuro de 12 horas, e alimentados com ração e água $a d$ libitum, durante cinco dias. Após o período de adaptação, todos os animais foram passaram por de jejum de 14 a 16 horas. Em seguida foram anestesiados com éter, e, amostras de sangue de $1,0 \mathrm{~mL}$ foram coletadas da artéria infraorbitária para determinar a glicemia - tempo zero (KING e GARNER, 1947). Em seguida, foi administrada a 16 ratos, $35 \mathrm{mg} / \mathrm{kg}$ de estreptozootocina diluída em tampão citrato de sódio ( $\mathrm{pH} 4,5)$, intravenosamente, no seio venoso do pênis, com os animais ainda anestesiados para indução do Diabetes mellitus. Os outros quatro ratos serviram como controle. Após cinco dias, coletou-se sangue, como anteriormente, para determinar a glicemia. Os animais que apresentaram hiperglicemia foram divididos em cinco grupos, com quatro ratos cada, que receberam tratamento oral uma vez por dia, com os diferentes extratos de Azadirachta indica, A. Juss. e com estreptozootocina em ultra alta diluições em sistemas dinamizados (estreptozootocina $6 \mathrm{CH}$ ).

Todos os animais foram tratados diariamente, por via oral $(0,2 \mathrm{~mL} / 100 \mathrm{~g}$ de animal), através de gavagem da seguinte forma: grupo controle, sem tratamento, só recebeu água; controle diabético, sem tratamento, só recebeu água; grupo tratado com água associado com extrato aquoso de Azadirachta indica, A. Juss. a 10\%; grupo tratado com água associado com extrato hidroalcoólico (70\%) de Azadirachta indica, A. Juss. a $10 \%$ e tratamento com água associado com estreptozootocina em ultra alta diluições em sistemas dinamizado (estreptozootocina $6 \mathrm{CH}$ ).

No $31^{\circ}$ dia, os animais foram eutanasiados e o coração inteiro foi removido da cavidade torácica e fixado em solução de Bouin durante 24 horas. Em seguida, o material foi desidratado em série crescente de álcool. As amostras foram recortadas, diafanizadas em benzol e inclusas em parafina. Após a microtomia semisseriada, a intervalos de $200 \mu \mathrm{m}$, os cortes histológicos à espessura de $5 \mu \mathrm{m}$ foram corados pela técnica de Hematoxilina e Eosina (HE) e PAS/Hematoxilina (TOLOSA et al., 2003) e fotomicrografados utilizando fotomicroscópio (LEICA 
DM 5000 B). Para cada animal foi confeccionada uma lâmina com cinco cortes histológicos, somando-se assim, 20 cortes histológicos para cada grupo experimental. As análises citoplasmáticas e cariométricas foram realizadas nas fibras musculares estriadas cardíacas transversais, medindo-se a área $\left(\mu \mathrm{m}^{2}\right)$, perímetro $(\mu \mathrm{m})$, diâmetro máximo $(\mu \mathrm{m})$, diâmetro mínimo $(\mu \mathrm{m})$ e o fator de forma (FF). O FF, bem como outros parâmetros foram analisados em panorâmica no programa Image Pro-Plus 4.0, Media Cybernetics Brazil, SP. O menor valor deste fator é igual a 1 , significando que a forma do citoplasma e/ou do núcleo se assemelha à forma de um círculo. Quando este fator for maior que a unidade, entende-se que a forma do citoplasma e/ou do núcleo não é circular.

$\mathrm{O}$ experimento foi realizado conforme $\mathrm{O}$ delineamento inteiramente casualizado com cinco tratamentos e quatro repetições e a análise de variância foi avaliada mediante procedimentos GLM do programa computacional Statistical Analysis Systems (SAS, 1999). As médias foram obtidas por meio do teste de Tukey a $5 \%$ de probabilidade (PIMENTEL GOMES, 2000).

\section{RESULTADOS E DISCUSSÃO}

\section{Histologia do coração}

As características histológicas do coração de ratos machos adultos Wistar estão ilustradas nas Figuras de 1 a 7. Os cortes histológicos desse órgão, em todos os grupos experimentais, apresentaram células musculares cardíacas com um ou dois núcleos centralmente localizados e com pequena quantidade de glicogênio. Além dos cardiomiócitos, verificamos a presença das fibras de Purkinje, as quais foram perceptivelmente maiores comparadas aos cardiomiócitos e revelaram quantidade significativa de glicogênio em um ou dois núcleos centralizados, à semelhança de Jacob et al. (1999) e Gomes et al. (2008)

O músculo estriado cardíaco contém numerosas mitocôndrias que ocupam, aproximadamente, $40 \%$ do volume citoplasmático, refletindo intenso metabolismo aeróbio desse tecido. Por outro lado, em outro trabalho, utilizando Microscopia Eletrônica de Transmissão (GOMES et al., 2008), os autores verificaram nos cardiomiócitos de ratos Wistar diabéticos aloxânicos, modificações nas mitocôndrias do grupo diabético, que foram aparentemente maiores e com numerosas cristas, quando comparadas ao controle sem nenhum tipo de tratamento. Essas alterações mitocondriais são devidas ao estresse oxidativo que o Diabetes mellitus ocasiona (SHEN et al., 2004). Desta forma, é plausível aventar a hipótese de que os ratos diabéticos não apresentaram alterações dessas organelas durante o período experimental havendo, portanto, necessidade de estudos duradouros e ulteriores, em casos desta enfermidade. Esses mesmos autores afirmararam que, em caso de hipoinsulinemia, ocorre deposição de glicogênio no miocárdio para poupar sua reserva em necessidades momentâneas (SHEN et al., 2004). Portanto, a maior intensidade da reação de PAS no miocárdio de ratos diabéticos pode ser decorrente ao glicogênio preservado nessa musculatura. Esse acúmulo pode ser maléfico para o órgão (JACOB et al.,1999) pois a presença do glicogênio pode causar a enfermidade de Pompe. A cardiomegalia, hipotonia muscular (acúmulo de glicogênio na musculatura e no sistema nervoso) e insuficiência cardíaca são sintomas possíveis desse acúmulo.

Com alusão ao glicogênio cardíaco, os cardiomiócitos (observado em todas as figuras) dos animais apresentaram afinidade tintorial ao método histoquímico do PAS/Hematoxilina, observada pela coloração magenta do glicogênio, comum em polissacarídeos, enquanto os núcleos celulares foram contracorados de azul pela hematoxilina. Como o glicogênio é reserva energética citoplasmática de disponibilidade imediata, quando há anormalidade no seu metabolismo ou no da glicose, são observados depósitos glicogênicos em excesso na forma de vacúolos claros no citoplasma, conferindo tom rosa-violeta a este metabólito na musculatura estriada cardíaca (KUMAR et al., 2010).

Os tratamentos com extrato aquoso de Azadirachta indica, A. Juss. a $10 \%$, extrato hidroalcoólico (70\%) de Azadirachta indica, A. Juss. a $10 \%$, e estreptozootocina $6 \mathrm{CH}$ foram semelhantes à coloração rosa-violeta evidenciada nos cardiomiócitos do grupo diabético, entretanto, mostraram coloração mais intensa que aos cardiomiócitos do grupo controle, corroborando com as afirmações de Jacob et al. (1999) e Gomes et al. (2008) e contrariando as afirmações de Satyanarayana et al. (2015).

\section{Morfometria}

Os parâmetros de diâmetro máximo $(\mu \mathrm{m})$ e área $\left(\mu \mathrm{m}^{2}\right)$ indicada na Tabela 1 revelaram que houve diferença $(p<0,05)$ entre os tratamentos controle; diabético; extrato aquoso de Azadirachta indica, A. Juss. a 10\%; extrato hidroalcoólico (70\%) de Azadirachta indica, A. Juss. a $10 \%$ e estreptozootocina, $6 \mathrm{CH})$. O perímetro $(\mu \mathrm{m})$, por sua vez, não diferiu significativamente $(p>0,05)$ entre os tratamentos.

Em relação à área, observamos diferença significativa na comparação entre o grupo controle e estreptozootocina $(6 \mathrm{CH})(\mathrm{p}<0,05)$. Os demais grupos experimentais não diferiram entre $\mathrm{si},(\mathrm{p}>0,05)$. No caso do diâmetro máximo foi observada diferença $(\mathrm{p}<0,05)$, na comparação entre o grupo controle e o tratado com extrato aquoso (70\%) de Azadirachta indica, A. Juss. a $10 \%$ e grupo estreptozootocina $(6 \mathrm{CH})$. Os demais grupos experimentais tratados não diferiram entre si, $(\mathrm{p}>0,05)$. Com referência ao perímetro, diâmetro mínimo e ao fator forma, não houve diferença $(p>0,05)$ entre os grupos controles e os demais grupos experimentais.

O grupo controle apresentou os maiores valores médios na maioria dos parâmetros analisados no citoplasma, comparado aos demais grupos experimentais, excetuando-se o FF cujo maior valor médio foi encontrado no grupo tratado com extrato hidroalcoólico (70\%) de Azadirachta indica, A. Juss. a 10\%. 


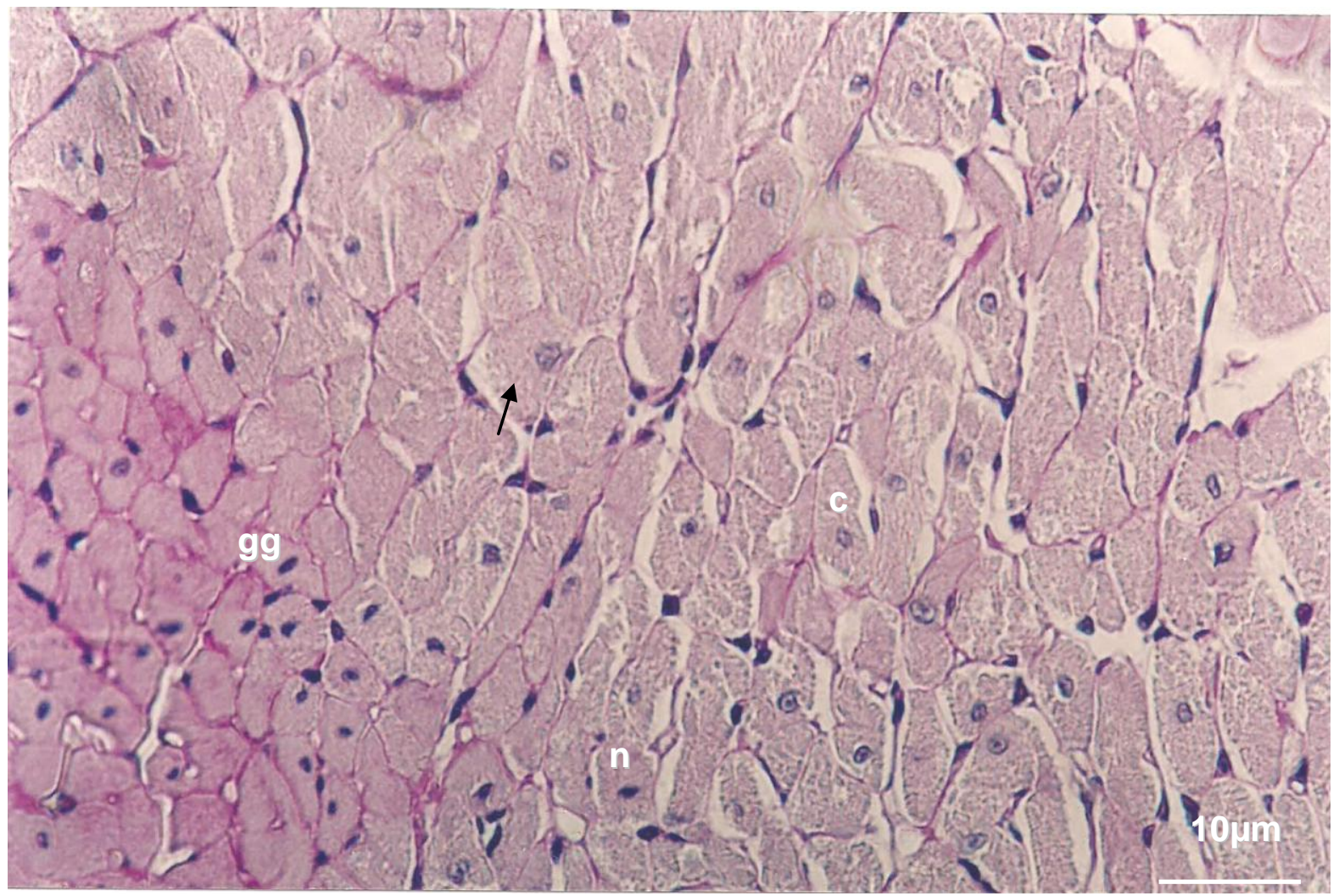

FIGURA 1 - Fotomicrografia, em corte transversal, do coração de rato macho albino Wistar, do grupo controle, indicando: grânulos de glicogênio $(\rightarrow)$ corados em magenta no citoplasma $(\mathbf{c})$, núcleo contracorado em azul $(\mathbf{n})$ e fibras de Purkinje ricas em glicogênio (gg). PAS/Hematoxilina, 40X.

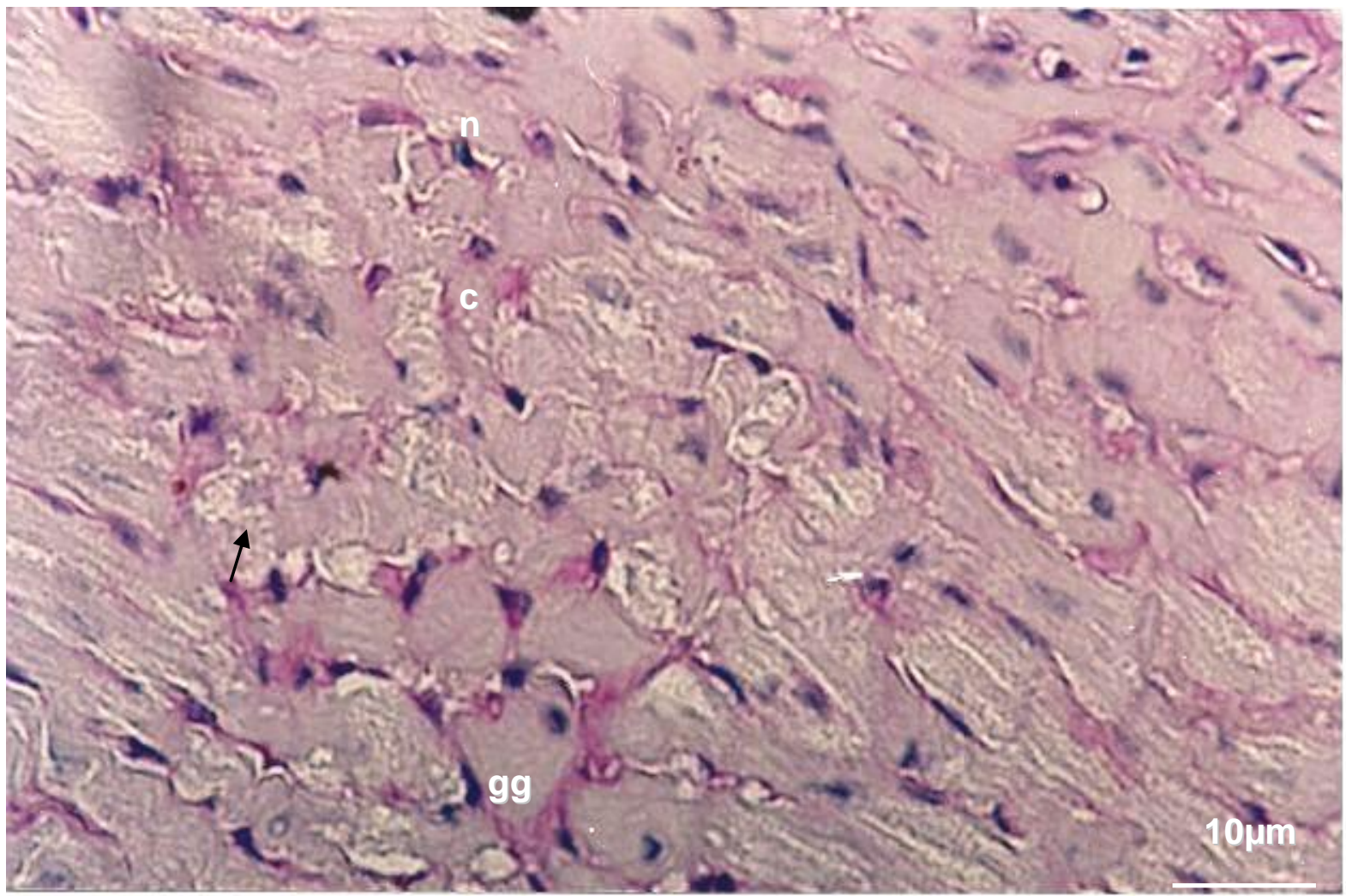

FIGURA 2 - Fotomicrografia, em corte transversal, do coração de rato macho albino Wistar, grânulos de glicogênio $(\rightarrow)$ corados em magenta no citoplasma (c), núcleo contracorado em azul (n) e fibras de Purkinje ricas em glicogênio (gg). PAS/Hematoxilina, 40X. 


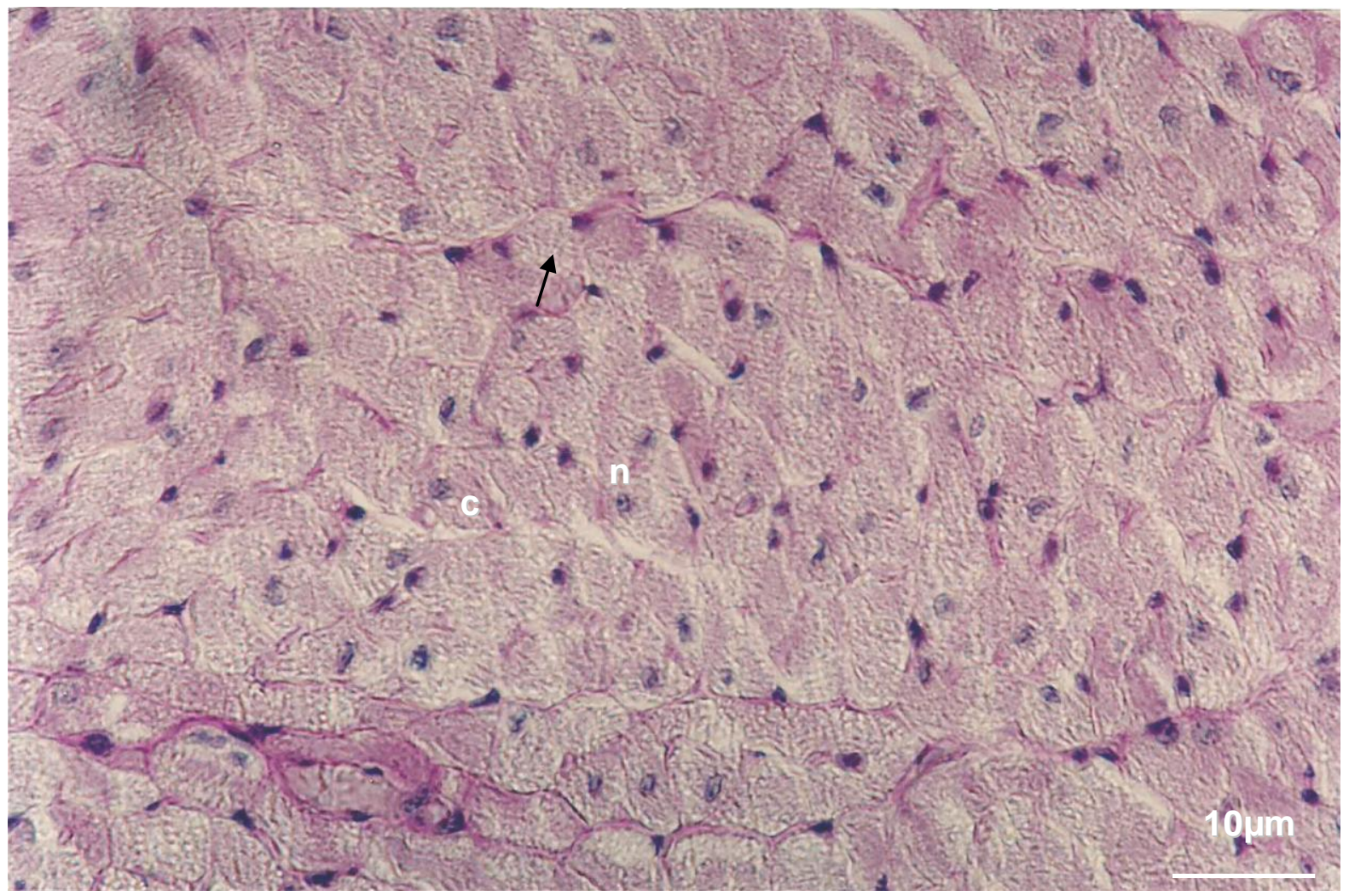

FIGURA 3 - Fotomicrografia, em corte transversal, do coração de rato macho albino Wistar, do grupo tratado com extrato aquoso de Azadirachta indica, A. Juss a 10\%, indicando: grânulos de glicogênio $(\rightarrow$ ) corados em magenta no citoplasma (c) e núcleo contracorado em azul (n). PAS/Hematoxilina, 40X.

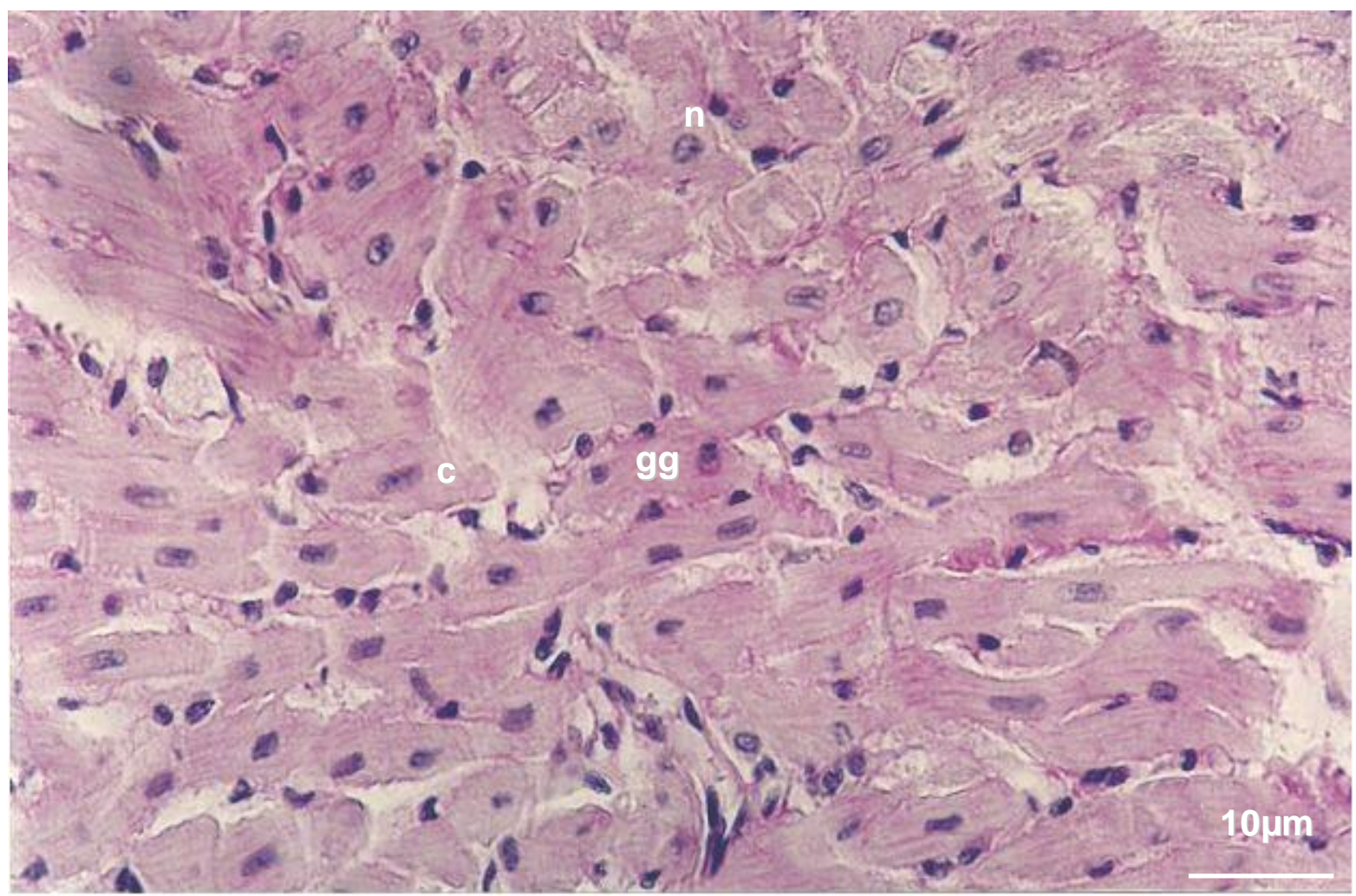

FIGURA 4 - Fotomicrografia, em corte transversal e longitudinal, do coração de rato macho albino Wistar, do grupo tratado com extrato aquoso de Azadirachta indica, A. Juss a 10\%, indicando: citoplasma (c) rico em grânulos de glicogênio (gg) corados em magenta nas fibras de Purkinje em azul e núcleo contracorado em azul (n). PAS/Hematoxilina, 40X. 


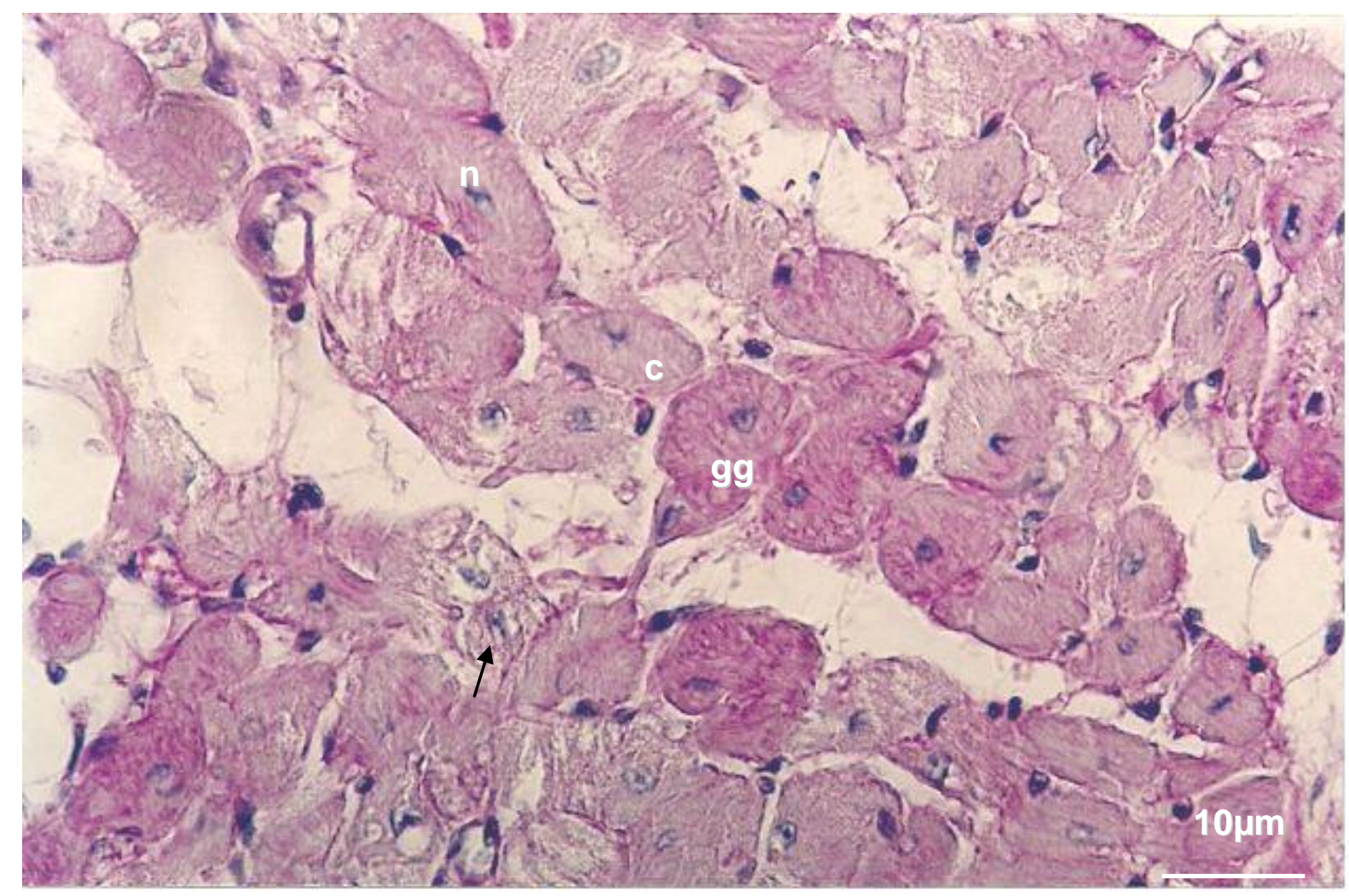

FIGURA 5 - Fotomicrografia, em corte transversal, do coração de rato macho albino Wistar, do grupo tratado com extrato hidroalcoólico (70\%) de Azadirachta indica, A. Juss a 10\%, indicando: grânulos de glicogênio $(\rightarrow)$ corados em magenta no citoplasma (c) dos cardiomiócitos e fibras de Purkinje ricas em glicogênio (gg) e núcleo contracorado em azul (n). PAS/Hematoxilina, 40X.

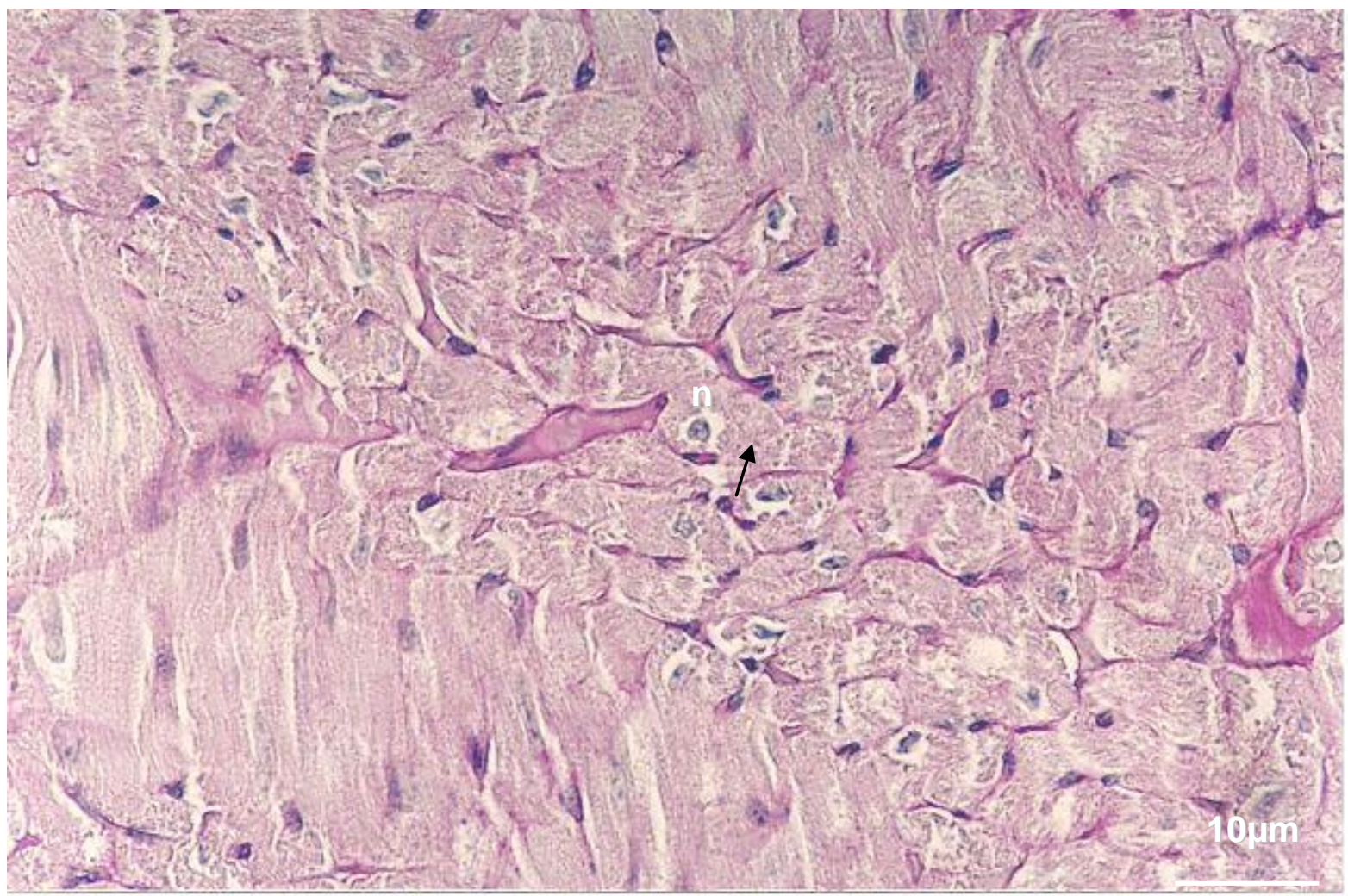

FIGURA 6 - Fotomicrografia, em corte transversal e longitudinal, do coração de rato macho albino Wistar, do grupo tratado com extrato hidroalcoólico (70\%) de Azadirachta indica, A. Juss a 10\%, indicando: grânulos de glicogênio $(\rightarrow)$ corados em magenta e núcleo contracorado em azul (n). PAS/Hematoxilina, 40X. 


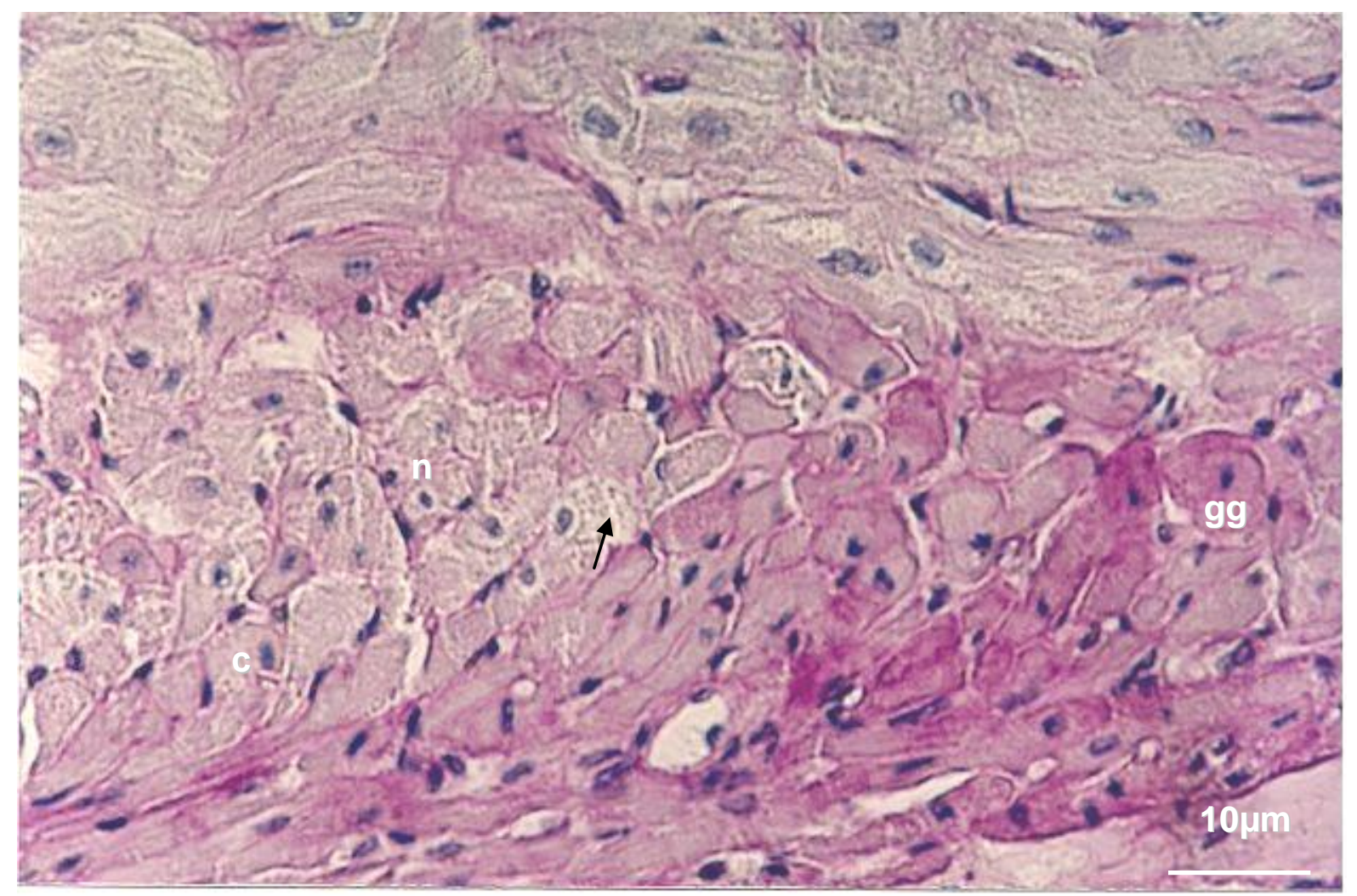

FIGURA 7 - Fotomicrografia, em corte transversal e longitudinal, do coração de rato macho albino Wistar, do grupo tratado com estreptozootocina, indicando: grânulos de glicogênio $(\rightarrow)$ corados em magenta no citoplasma $(\mathbf{c})$ dos cardiomiócito e fibras de Purkinje ricas em glicogênio (gg) e núcleo contracorado em azul (n). PAS/Hematoxilina, 40X.

Tabela 1 - Médias e desvio padrão dos parâmetros morfométricos do citoplasma de cardiomiócitos de ratos machos albinos Wistar dos grupos controle e diabéticos tratados com extrato aquoso e hidroalcoólico de A. indica, A. Juss. e estreptozootocina $6 \mathrm{CH}$.

\begin{tabular}{lccccc}
\hline Tratamento & Área $\left(\boldsymbol{\mu m}^{2}\right)^{*}$ & Perímetro $(\boldsymbol{\mu m})$ & $\begin{array}{c}\text { Diâmetro } \\
\text { máximo } \\
(\boldsymbol{\mu m}) * *\end{array}$ & $\begin{array}{c}\text { Diâmetro } \\
\text { mínimo } \\
(\boldsymbol{\mu m})\end{array}$ & $\begin{array}{c}\text { Fator } \\
\text { Forma }\end{array}$ \\
\hline Controle & $205,64 \pm 19,35^{\dagger}$ & $46,09 \pm 2,85^{\dagger}$ & $16,28 \pm 1,04^{\dagger}$ & $10,02 \pm 1,38^{\dagger}$ & $0,84 \pm 0,038^{\dagger}$ \\
Diabético & $190,51 \pm 17,93^{\dagger \dagger}$ & $42,98 \pm 2,66^{\dagger}$ & $14,60 \pm 0,93^{\dagger *}$ & $8,26 \pm 1,14^{\dagger}$ & $0,78 \pm 0,035^{\dagger}$ \\
Extrato Aquoso & $171,35 \pm 16,12^{\dagger \dagger}$ & $41,20 \pm 2,55^{\dagger}$ & $13,99 \pm 0,89^{\ddagger}$ & $7,96 \pm 1,09^{\dagger}$ & $0,80 \pm 0,036^{\dagger}$ \\
Extr. Hidroalcóolico & $178,37 \pm 16,78^{\dagger \dagger}$ & $43,19 \pm 2,67^{\dagger}$ & $14,38 \pm 0,91^{\dagger *}$ & $8,33 \pm 1,15^{\dagger}$ & $0,85 \pm 0,038^{\dagger}$ \\
Estreptozootocina 6CH & $167,56 \pm 15,77^{\dagger}$ & $41,67 \pm 2,58^{\dagger}$ & $13,99 \pm 0,89^{\ddagger}$ & $8,80 \pm 1,21^{\dagger}$ & $0,84 \pm 0,038^{\dagger}$ \\
\hline
\end{tabular}

Médias seguidas de símbolos diferentes nas colunas diferem significativamente entre si pelo Teste de Tukey a $1 \%$ e $5 \%$ de probabilidade. $P<0,05 ; * * P<0,01$

Tabela 2 - Médias e desvio padrão dos parâmetros morfométricos no núcleo de cardiomiócitos de ratos machos albinos Wistar dos grupos controle e diabéticos tratados com extrato aquoso e hidroalcoólico de A. indica, A. Juss. e estreptozootocina $6 \mathrm{CH}$

\begin{tabular}{|c|c|c|c|c|c|}
\hline Tratamento & Área $\left(\mu \mathrm{m}^{2}\right)^{*}$ & Perímetro $(\mu \mathrm{m})$ & $\begin{array}{c}\text { Diâmetro } \\
\text { máximo } \\
(\mu \mathrm{m}) * *\end{array}$ & $\begin{array}{c}\text { Diâmetro } \\
\text { mínimo } \\
(\mu \mathrm{m})\end{array}$ & $\begin{array}{l}\text { Fator } \\
\text { Forma }\end{array}$ \\
\hline Controle & $13,96 \pm 2,84^{\dagger}$ & $14,17 \pm 1,39^{\dagger}$ & $4,42 \pm 0,33^{\dagger}$ & $3,34 \pm 0,40^{\dagger}$ & $1,20 \pm 0,06^{\dagger}$ \\
\hline Diabético & $13,43 \pm 2,73^{\dagger}$ & $14,02 \pm 1,37^{\dagger}$ & $4,43 \pm 0,33^{\dagger}$ & $3,36 \pm 0,40^{\dagger}$ & $1,22 \pm 0,06^{\dagger}$ \\
\hline Extrato Aquoso & $11,02 \pm 2,24^{\dagger}$ & $12,68 \pm 1,24^{\dagger}$ & $3,84 \pm 0,28^{\dagger \dagger}$ & $2,68 \pm 0,32^{\dagger}$ & $1,23 \pm 0,06^{\dagger}$ \\
\hline Extr. Hidroalcóolico & $9,48 \pm 1,93^{\dagger}$ & $11,66 \pm 1,14^{\dagger}$ & $3,62 \pm 0,27^{\ddagger}$ & $2,70 \pm 0,33^{\dagger}$ & $1,18 \pm 0,06^{\dagger}$ \\
\hline Estreptozootocina $6 \mathrm{CH}$ & $9,80 \pm 1,99^{*}$ & $11,87 \pm 1,16^{\dagger}$ & $3,75 \pm 0,28$ & $2,72 \pm 0,33^{\dagger}$ & $1,11 \pm 0,06^{\dagger}$ \\
\hline
\end{tabular}

Médias seguidas de símbolos diferentes nas colunas diferem significativamente entre si pelo Teste de Tukey a $1 \%$ e $5 \%$ de probabilidade. $P<0,05$; $* * P<0,01$ 
Os resultados indicados na Tabela 2, referentes aos parâmetros mensurados no núcleo dos cardiomiócitos de ratos machos albinos Wistar, revelaram que houve diferenças significativas entre os tratamentos (controle, diabético, grupo tratado com extrato aquoso de Azadirachta indica, A. Juss. a 10\%, extrato hidroalcoólico (70\%) de Azadirachta indica, A. Juss. a $10 \%$ e grupo tratado com estreptozootocina $(6 \mathrm{CH})$. Estas diferenças foram avaliadas nas probabilidades de $1 \%$ para o diâmetro máximo $(\mu \mathrm{m})$ e de $5 \%$ para a área $\left(\mu \mathrm{m}^{2}\right)$, perímetro $(\mu \mathrm{m})$ e diâmetro mínimo $(\mu \mathrm{m})$. Porém, o fator forma não diferiu $(p>0,05)$ entre os tratamentos. Comprovaram-se diferenças $(p<0,05)$ entre os valores médios do diâmetro máximo no controle e diabético com os grupos tratados com extrato hidroalcoólico $(70 \%)$ de Azadirachta indica, A. Juss. a $10 \%$ e estreptozootocina $6 \mathrm{CH}$. Os demais grupos experimentais tratados não diferiram entre $s i,(p>0,05)$.

O grupo controle apresentou, na maioria dos parâmetros analisados no núcleo dos cardiomiócitos, os maiores valores médios, excetuando-se os diâmetros máximo e mínimo, cujos maiores valores foram encontrados no controle diabético, assim como o FF que apresentou o maior valor médio no grupo tratado com extrato aquoso (70\%) de Azadirachta indica, A. Juss. a $10 \%$.

Os resultados morfométricos revelaram que o grupo sem nenhum tratamento prévio, apresentou os maiores valores para a maioria dos parâmetros analisados no citoplasma e núcleo dos cardiomiócitos. Sugere-se que os tratamentos não favoreceram a contratilidade cardíaca, diferente de Silva et al. (2006) que observaram maior amplitude de contratilidade e aumento do volume de cardiomiócitos em ratos Wistar diabéticos que praticaram natação.

Desta forma, acreditamos que o tratamento fitoterápico mediante o uso do extrato aquoso de Azadirachta indica, A. Juss. a $10 \%$ e do extrato hidroalcoólico (70\%) de Azadirachta indica, A. Juss. a $10 \%$ não proporcionou modificações nas dimensões dos parâmetros analisados no citoplasma e núcleo dos cardiomiócitos. O extrato aquoso de Azadirachta indica, A. Juss. a $10 \%$ não aumentou as dimensões do citoplasma e núcleo destas células quando comparado ao controle diabético, excetuando-se o fator forma que, provavelmente, não interfere nas dimensões celulares. O extrato hidroalcoólico (70\%) de Azadirachta indica, A. Juss. a $10 \%$ aumentou discretamente apenas o perímetro, diâmetro mínimo e o FF do citoplasma dos cardiomiócitos. Em relação ao núcleo, o nim não promoveu aumento nos parâmetros analisados. No que diz respeito ao tratamento homeopático com estreptozootocina $(6 \mathrm{CH})$, também notamos que os valores médios, na maioria dos parâmetros analisados no citoplasma e núcleo dos cardiomiócitos foram inferiores ao grupo diabético, excetuando-se apenas o diâmetro mínimo e o fator de forma do citoplasma.

\section{CONCLUSÃO}

A Azadirachta indica, A. Juss. aclimatada no Brasil e o tratamento homeopático tanto com o extrato aquoso como o hidroalcoólico de estreptozootocina $(6 \mathrm{CH})$ utilizados em ratos diabéticos induzidos pela estreptozootocina não modificam o perímetro celular e as reservas de glicogênio nos cardiomiócitos. Além disso, o extrato líquido ou hidroalcóolico proporcionam resultados semelhantes na histologia cardíaca. A manutenção das dimensões das fibras musculares estriadas cardíacas demonstra que o fitoterápico com Azadirachta indica A. Juss. aclimatada no Brasil e homeopático com estreptozootocina $(6 \mathrm{CH})$ utilizados no Diabetes mellitus não interferem na histomorfometria cardíaca em um período de 30 dias de tratamento. Estudos futuros e com tempo maior de tratamento, possivelmente permitirão detectar alterações nos cardiomiócitos, utilizando-se técnicas mais acuradas, tais como microscopia eletrônica de transmissão ou confocal.

\section{AGRADECIMENTOS}

Os autores agradecem a concessão da bolsa de Iniciação Científica (2011/00121-7) e do apoio financeiro concedido pela FAPESP - Fundação de Amparo à Pesquisa do Estado de São Paulo.

\section{REFERÊNCIAS}

BRASIL. R. B. Aspectos botânicos, usos tradicionais e potencialidades de Azadirachta indica (NEEM. Enciclopédia Biosfera, Centro Científico Conhecer Goiânia, v.9, n.17, 2013.

DING, A.; RODRIGUES, B. Role of changes in cardiac metabolism in development of diabetic cardiomyopathy. American Journal of Physiology Heart Circulatory Physiology, v. 291, p. 1489-506, 2006.

GOMES, R. J.; LEME, J. A. C. A.; MELLO, M. A. R.; LUCIANO, E.; CAETANO, F. H. Efeitos do treinamento de natação em aspectos metabólicos e morfológicos de ratos diabéticos. Motriz, v.14, p. 320-328, 2008.

JACOB, J. L. B.; LEANDRO, R. L.; PARRO, A. J. Doença de Pompe ou Glicogenose Tipo IIa. Arquivo Brasileiro de Cardiologia, v. 76, n. 6, p. 435-437, 1999.

KING, E. J.; GARNER, R. J. Colorimetric determination of glucose. Journal of Clinical Pathology v. 1, n.1, p. 3033, 1947.

Kumar, V.; Abbas, A.K.; Fausto, N. Robins e Cotran: Patologia - Bases Patológicas das doenças. 8. ed., Rio de Janeiro: Elsevier; 2010.1480p

MOSSINI, S. A. G.; KEMMELMEIER, C. A árvore Nim (Azadirachta indica A. Juss): Múltiplos Usos. Acta farmacéutica bonaerense, v. 24, p. 139-148, 2005.

PIMENTEL GOMES, F. Curso de estatística experimental. Piracicaba (SP). Nobel, v.14, p. 477, 2000.

SATYANARAYANA, K.; SRAVANTHI, K.; ANAND SHAKER, I.; PONNULAKSHMI, R. 
Molecular approach to identify antidiabetic potential of Azadirachta indica. Journal Ayurveda Integrative Medicine, v.6, n.3, p. 165-174, 2015.

SAXENA, R. C. Scope of Nim developing countries. In: World Nim conference Souvenir-Bengalore; Proceedings. Nairobe, p. 24-28, 1998

SHEN, X.; ZHANG, K.; KAUFMAN, R. The unfolded protein response - a stress signaling pathway of the endoplasmic reticulum. Journal Chemical Neuroanatomy, v.28 n.1-2, p. 79-92, 2004.

SILVA, R. F.; LOPES, R. A.; SALA, M. A.; VINHA, D.; REGALO, S. C. H.; SOUZA, A. M.; GREGÓRIO, Z. M. O. Action of trivalent chromium on rat liver structure. Histometric and haematological studies. Internal Journal Morphology, v.24 n.2, p. 197-203, 2006.

SILVA, M. F.; PELÚZIO, M. C. G.; AMORIM, P. R. S.; LAVORATO, V. N.; SANTOS N. P.; BOZI, L. H. M.; ARLETE RITA PENITENTE, A. R. P; FALKOSKI, D. L.; BERFORT F. G.;NATALI, A. J. Treinamento em Natação Atenua a Disfunção Contrátil de Cardiomiócitos de Ratos Diabéticos. Dísponível em: http://www.scielo.br/pdf/abc/v97n1/aop04611. Acesso em: $22 / 10 / 2015$
STATISTICAL ANALISYS SYTEMS INSTITUTE INC. SAS User's Guide: Statistics (version 5 edn). SAS Institute. INC., Cary, NC, 200, 1999.

TOLOSA, E. M. C.; RODRIGUES, C. J.; BEHMER, A. O.; FREITAS-NETO, A. G. Manual de técnicas para histologia normal e patológica. $2^{a}$ ed. São Paulo (SP): Manole, p. 331, 2003.

VILELA, J. A. R. Efeito da utilização de Óleo de nim (Azadirachta indica) por via Dérmica e da Moxidectina por via Subcutânea na Prevenção de Infestação por Dermatobia hominis (LINNAEUS JR., 1781) (DIPTERA; CUTEREBRIDAE) em Bovinos. Dissertação de Mestrado. Universidade Federal rural do Rio de Janeiro. Instituto de Veterinária. Curso de Pós-Graduação em Ciências Veterinárias, p. 53, 2008. 